\title{
RÉCENTS PROGRÈS DANS LA FABRICATION DE JONCTIONS JOSEPHSON. UTILISATION DE NIOBIUM OBTENU PAR PULVÉRISATION CATHODIQUE
}

\author{
P. CARDINNE, B. MANHES \\ L'Air Liquide, Centre d'Etudes Cryogéniques, B. P. 15, 38-Sassenage, France \\ et
}

J. E. NORDMAN

Université du Wisconsin, USA

\begin{abstract}
Résumé. - Les technologies de fabrication de jonctions tunnel niobium-oxyde de niobiumplomb et niobium-germanium-plomb, sont exposées. Le film de niobium est déposé par pulvérisation cathodique RF puis soumis à un décapage ionique (back-sputtering) opération qui est nécessaire pour restaurer les propriétés intrinsèques du matériau et obtenir de bonnes caractéristiques tunnel. Cependant, cette opération modifie la couche superficielle du niobium, ce qui se traduit essentiellement par l'apparition pour $V>\Delta \mathrm{Pb}+\Delta \mathrm{Nb}$ d'une résistance négative.

Nous proposons un modèle faisant état d'une couche de matériau à température critique réduite. Cependant, à l'heure actuelle, ce modèle ne rend pas compte de tous les faits expérimentaux. Les jonctions obtenues ont de bonnes caractéristiques tunnel et une bonne stabilité dans le temps.

Abstract. - Techniques for fabrication of tunnel junctions of niobium-niobium oxide-lead and niobium-germanium-lead are described. The niobium film is deposited by RF sputtering and is subsequently sputter etched, an operation which is necessary to produce good tunneling characteristics. This operation modifies the niobium surface as evidenced by the appearance of a negative resistance region for $V>\Delta \mathrm{Pb}+\Delta \mathrm{Nb}$.

Although a model assuming a thin surface layer with reduced transition temperature is reasonable, it does not explain all the experimental facts. The junctions obtained have good tunneling characteristics and are quite stable in time.
\end{abstract}

Introduction. - L'utilisation de films en niobium présente par rapport aux films de plomb des avantages indiscutables en ce qui concerne la solidité et la durée de vie des jonctions tunnel.

Nous avons donc travaillé en vue de remplacer dans les jonctions supraconducteur-semiconducteursupraconducteur le premier film par un film de niobium [1]. Il est en effet technologiquement difficile, pour des raisons que nous exposerons plus loin, d'élaborer des jonctions dont les deux électrodes sont en niobium.

Dans cet exposé nous parlerons donc essentiellement de la technologie employée pour obtenir des jonctions niobium-oxyde de niobium-plomb, qui constituent une première étape, puis des jonctions niobiumgermanium-plomb.

Nous exposerons les résultats et tenterons de donner une interprétation des phénomènes rencontrés.

1. Conditions expérimentales. - 1.1 CARACTÉRISTIQUES DE POMPAGE - Le prévidage de l'enceinte s'effectue avec une pompe sèche puis avec une pompe à zéolithes qui permet d'atteindre une pression de quelque $10^{-3}$ torr. Ensuite le vide secondaire est obtenu à l'aide d'une cryopompe à hélium liquide (la pression est, moyennant certaines précautions, de l'ordre de $10^{-7}$ torr).

Avant opération il est nécessaire de procéder à un étuvage très soigné non seulement de la cloche en pyrex mais surtout des parties métalliques qui seront ultérieurement chauffées par la décharge. Ce point est très important et conditionne directement les qualités des films de niobium obtenus. C'est ainsi que nous devons étuver toute l'anode porte-substrats à une température de $350^{\circ} \mathrm{C}$ pendant plusieurs heures.

Ensuite les conditions de fonctionnement choisies sont les suivantes :

- Flux d'argon $\simeq 0,1 \mathrm{Tl} / \mathrm{s}$.

- Pression dans la cloche $\simeq 4 \times 10^{-3}$ torr.

Notons l'intérêt que l'on a, dans cette expérience, à utiliser une cryopompe ; en effet, on peut faire varier dans une grande gamme la vitesse de pompage de l'appareil, ce qui permet ainsi de choisir la valeur du flux d'argon avec une grande souplesse.

1.2 ENSEMBle DE PUlVÉRISATION CATHOdiQue. Nous utilisons un module de pulvérisation cathodique radiofréquence bicathode, type PSP 24 Veeco. Les cathodes initialement prévues pour pouvoir utiliser 
des matériaux en grains sont maintenant en matériau massif, ce qui assure une meilleure reproductibilité.

Les masques utilisés étaient placés directement sur les substrats en verre, ceci a l'avantage d'obtenir des bords francs.

Les films de niobium sont évaporés en utilisant une puissance de $400 \mathrm{~W}$, la vitesse de dépôt est d'environ $160 \AA / \mathrm{min}$. Durant le dépôt le substrat est à la masse, il n'est pas refroidi et la décharge le porte à une température d'environ $80^{\circ} \mathrm{C}$.

Ces conditions permettent d'obtenir des films de niobium ayant des températures critiques comprises entre 8 et $8,6 \mathrm{~K}$ et des rapports de résistivité

$$
\rho=R_{300 \mathrm{~K}} / R_{77 \mathrm{~K}}
$$

compris entre 1,6 et 2 . Les épaisseurs des films sont de l'ordre de $1500 \AA$.

2. Elaboration de jonctions à barrière d'oxyde. Les films de niobium obtenus ont des largeurs d'environ $4 \mathrm{~mm}$. Nous avons choisi de partir de films larges et d'en délimiter ensuite la partie utile par un dépôt de $\mathrm{SiO}$. Bien que cela représente une opération supplémentaire, nous avons préféré la faire plutôt que d'utiliser des masques très étroits ce qui, en pulvérisation cathodique, pose souvent des problèmes de profil du dépôt. Il est en effet bien connu que l'on peut obtenir des profils présentant une vallée médiane lorsqu'on utilise dans ces conditions des masques larges de quelques dixièmes de millimètres.

Ainsi, après obtention du film de niobium :

1) Nous déposons deux bandes de $\mathrm{SiO}$ pour délimiter la partie centrale du film.

2) Nous procédons à un nettoyage ionique (backsputtering) de l'ensemble, sur lequel nous reviendrons plus tard.

3) Nous oxydons le film en atmosphère d'oxygène humide pendant quelques minutes à environ $130^{\circ} \mathrm{C}$.

4) Nous déposons par évaporation thermique sous un vide de l'ordre de $5 \times 10^{-6}$ torr un film de plomb transversalement au film de niobium. La largeur de ce film est de $0,2 \mathrm{~mm}$ ce qui détermine une surface de jonction de $0,04 \mathrm{~mm}^{2}$.

3. Résultats relatifs aux jonctions à barrière d'oxyde. - 3.1 REMARQUES PRÉLIMINAIRES - L'expérience a montré que pour obtenir de bonnes caractéristiques de jonction tunnel il est nécessaire de réunir deux conditions de base :

1) Avoir un film présentant des qualités supraconductrices suffisantes.

2) Nettoyer la surface de ces films par bombardement ionique.

Explicitons ces deux conditions :

1) Nous avons observé que lorsque le rapport de résistivité $\rho_{300 \mathrm{~K}} / \rho_{77 \mathrm{~K}} \mathrm{du}$ film de niobium est supérieur à 1,5 on obtient des caractéristiques «tunnel " correctes, et lorsque le rapport est inférieur à 1,5 les caractéristiques présentent toujours un fort courant de fuite. Ce critère est plus valable que celui qui consiste à mesurer la température critique. En effet, la température critique est liée à la nature des impuretés (il est par exemple bien connu que l'oxygène abaisse le $T_{\mathrm{c}}$, ce qui n'est pas le cas de $\mathrm{N}_{2}$ ) alors que le rapport de résistivité relié au libre parcours moyen donne une indication sur la pureté du matériau.

2) Nous savons, d'après la littérature, que la surface des films de niobium est souvent polluée ce qui produit une couche de métal soit normal soit à $T_{\mathrm{c}}$ réduit. Nous avons mis en évidence expérimentalement que cette couche doit être enlevée si l'on désire obtenir de bonnes caractéristiques tunnel [2].

En effet, si nous ne pratiquons pas ce nettoyage par bombardement ionique, nous obtenons des caractéristiques analogues à celles exposées figure 1 dans les-

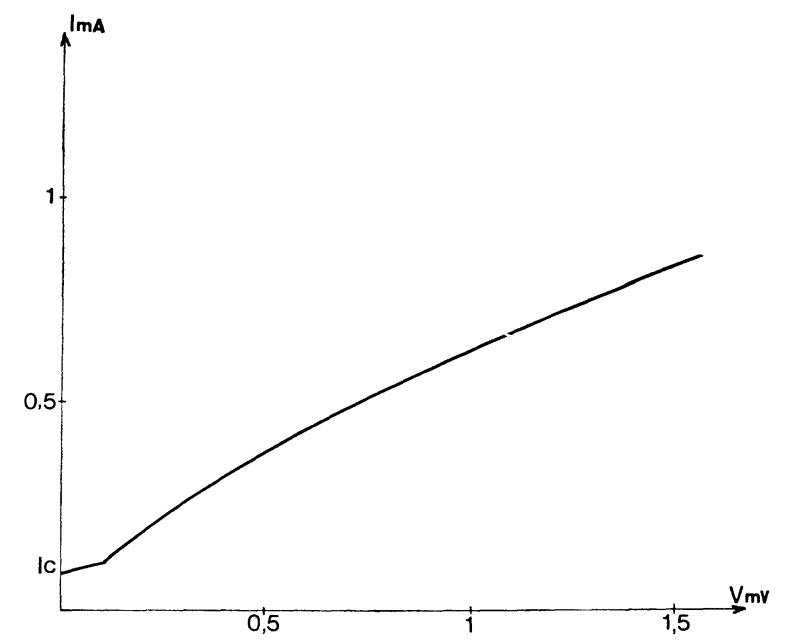

Fig. 1. - Caractéristique $I(V)$ typique d'une jonction niobium-oxyde de niobium-plomb n'ayant pas subi de nettoyage ionique.

quelles le courant de fuite est considérable. Par contre, si avec un film de niobium de même qualité nous procédons à un nettoyage ionique de quelques minutes à une puissance réduite $(100 \mathrm{~W})$ nous obtenons des caractéristiques analogues à celles de la figure 2 c'està-dire avec un faible taux de courant de fuite.

Ces remarques sont à rapprocher des résultats obtenus sur du niobium massif [3] pour lequel le nettoyage ionique s'est aussi avéré indispensable. Dans le cas des films ceci nous amène donc à penser que la couche endommagée provient d'une pollution gazeuse. Lorsque cette couche vient d'être élaborée et se refroidit, elle piège en surface des impuretés gazeuses de l'atmosphère résiduelle. Pour le cas des matériaux massifs la pollution provient vraisemblablement d'un désordre structurel amené par le polissage. Il est intéressant de noter que bien que les causes soient différentes le phénomène et la façon d'y remédier sont les mêmes.

3.2 CARACTÉRISTIQUES $I(V)$ DES JONCTIONS A OXYDE. - 3.2.1 Constatations expérimentales. - A partir des caractéristiques $I(V)$ obtenues telles que celles de la 
figure 2, nous pouvons résumer les faits expérimentaux de la façon suivante :

1) La valeur $V_{1}$ définie sur la figure 2 , comme correspondant à peu près à la somme des gaps $\Delta \mathrm{Pb}+\Delta \mathrm{Nb}$ varie entre 2,5 et $2,7 \mathrm{meV}$ (à $4,2^{\circ}$ ) pour des rapports de résistivité des films variant respectivement entre 1,6 et 1,9 .

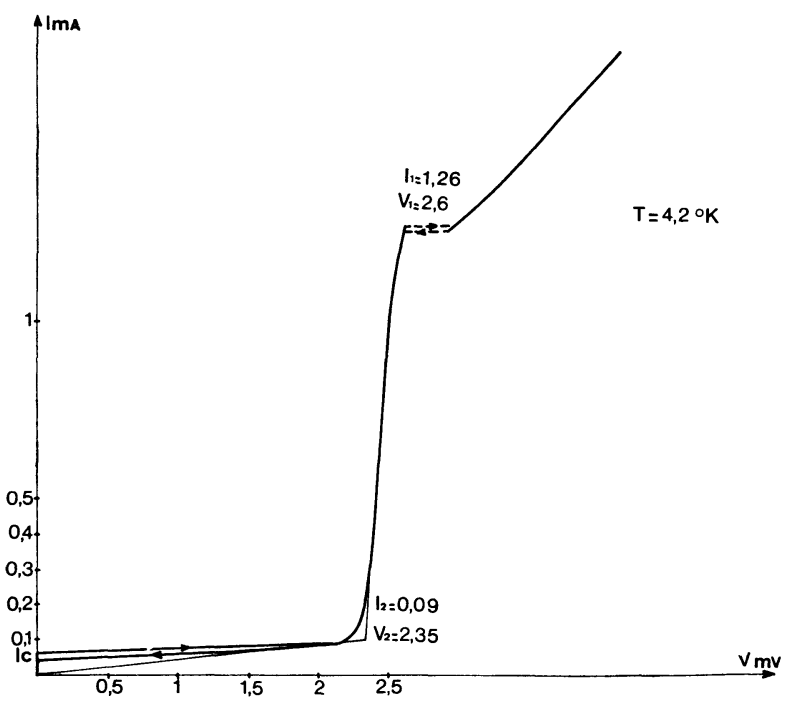

FIG. 2. - Caractéristique $I(V)$ de la jonction no 16 A niobiumoxyde de niobium-plomb.

2) Pour $v>\Delta \mathrm{Pb}+\Delta \mathrm{Nb}$, nous constatons l'existence d'une zone à résistance négative très accentuée.

3) La valeur $V_{2}$ définie sur la figure 2 et correspondant ainsi, d'après modèle de la figure 3 à peu près à $\Delta \mathrm{Pb}+\Delta \mathrm{N}(\Delta \mathrm{N}$ étant le gap de la couche superficielle) varie entre 2,2 et 2,5 . Cette variation ne peut être reliée à d'autres paramètres expérimentaux.

4) Le rapport entre les courants $I_{1}$ et $I_{2}$, qui correspond au taux de résistance de fuite, varie entre 10 et 15 .

3.2.2 Commentaires et interprétation. - Reprenons les points précédents :

1) Nous avons d'abord pensé que les variations de $V_{1}$ pouvaient être imputées aux variations de qualité des films et effectivement il y a une tendance dans ce sens, c'est-à-dire que les films de bonne qualité donnent lieu généralement à des $V_{1}$ plus élevés. Cependant les résultats obtenus avec un matériau massif [3] ont montré que la valeur de $V_{1}$ dépendait aussi du traitement ionique, ce que nous ne pouvons expliquer, donc nous restons prudents quant à une tentative visant à relier $V_{1}$ et $\rho$.

2) Signalons que l'effet de résistance négative pour $V>\Delta \mathrm{Pb}+\Delta \mathrm{Nb}$ peut être associé au schéma d'énergie donné figure 3 . En effet, l'existence d'une telle distribution avec un plus grand nombre d'états près du gap doit donner lieu, d'après [4] à une résistance négative.

Ce modèle suppose la présence, à la surface du niobium, d'une couche de matériau ayant une température critique inférieure à celle du niobium.

Ainsi, sur le plan théorique, cet effet a été prédit en utilisant la théorie de Mac Millan, mais sur le plan pratique il a été rarement observé [5], [6]. Il semble d'après [6] et nos travaux que la seule façon d'obtenir cet effet d'une façon reproductible et vraiment nette (à $4,2 \mathrm{~K}$ ) consiste à bombarder la surface avec des ions argon.

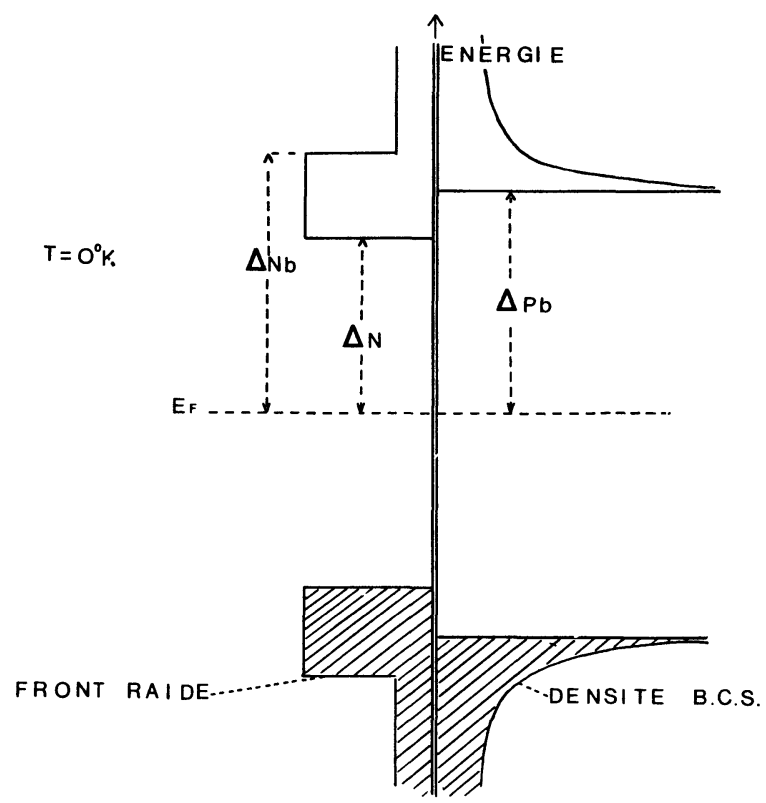

Fig. 3. - Modèle proposé des densités d'états en fonction de l'énergie pour le sandwich: niobium-niobium superficieloxyde de niobium-plomb.

Il est donc probable que ce bombardement crée, soit à cause de l'implantation d'ions argon, soit à cause d'un désordre structurel, une couche superficielle de propriétés différentes. A l'heure actuelle nous ne sommes pas en mesure de savoir si cette couche est faite d'un métal normal devenant supraconducteur par effet de proximité ou d'un matériau à $T_{\mathrm{c}}$ plus faible que celui du niobium. Nous pouvons seulement dire, du fait que le courant de fuite pour $V<\Delta \mathrm{Nb}+\Delta \mathrm{Pb}$ croît en général régulièrement à partir de $V=0$, qu'il est peu probable que la couche superficielle soit faite d'un matériau supraconducteur sans gap [7].

3) $\mathrm{La}$ valeur $V_{2} \simeq \Delta \mathrm{N}+\Delta \mathrm{Pb}$ correspond à la polarisation pour laquelle le bord inférieur de la bande interdite du plomb commence à tomber en face d'états vides. Si le courant augmente brusquement pour cette valeur, cela signifie qu'il existe bien une bande interdite comme représente la figure 3 , la valeur de $V_{2}$ devrait donc permettre de calculer la valeur du gap correspondant à cette bande interdite.

Toutefois, la situation expérimentale n'est pas parfaitement claire et en particulier, le fait que $V_{2}$ varie d'un échantillon à l'autre n'est pas explicable puisque le traitement de nettoyage ionique est toujours le même $(100 \mathrm{~W}, 2 \mathrm{~min})$. Notons cependant que la façon 
dont nous mesurons $V_{2}$ la rend dépendante du taux de courant de fuite, mais il est cependant possible d'obtenir des valeurs $V_{2}$ très faible, ceci pour un taux de résistance élevé (supérieur à 12).

4. Dépendance en fonction du champ magnétique. La dépendance du courant Josephson en fonction du champ magnétique n'a jamais été trouvée excellente. Nous avons souvent observé une figure d'interférences soit entre deux, soit entre plus de deux jonctions.

Ceci nous amène à penser que la barrière n'est pas très uniforme.

5. Jonctions à barrière de germanium. - Nous avons encore assez peu de résultats en ce qui concerne ce type de jonctions.

La procédure suivie est exactement la même que précédemment à l'exception du fait que nous déposons une couche de germanium après avoir nettoyé la surface du film par bombardement ionique et avant une oxydation rendue nécessaire pour éviter les courtcircuits.

Le matériau est déposé par pulvérisation cathodique, la cathode en germanium massif est constituée de plusieurs monocristaux disposés en mosaïque (la résistivité du matériau de départ est de l'ordre de $20 \Omega . \mathrm{cm}$ ).

La résistivité en film mince du matériau déposé est de l'ordre de $0,6 \Omega$.cm à $300 \mathrm{~K}$, et varie de deux ordres de grandeurs entre la température ambiante et $77 \mathrm{~K}$.

La couche déposée pour constituer la barrière est épaisse d'environ $100 \AA ̊$.

Les conditions de dépôt sont les suivantes :

$$
\begin{array}{ll}
\text { Puissance } & \simeq 50 \mathrm{~W} \\
\text { Durée } & 10 \mathrm{~s} \\
\text { Pression } & \simeq 4 \times 10^{-3} .
\end{array}
$$

Le substrat est refroidi pendant le dépôt.

En ce qui concerne les caractéristiques $I(V)$, nous pouvons dire pour le moment que nous retrouvons tous les traits précédemment définis et relatifs aux jonctions à barrière d'oxyde. Actuellement, le seul fait qui permet de distinguer un type de jonction de l'autre à partir des caractéristiques $I(V)$ est une variation importante de l'impédance en fonction de la température.

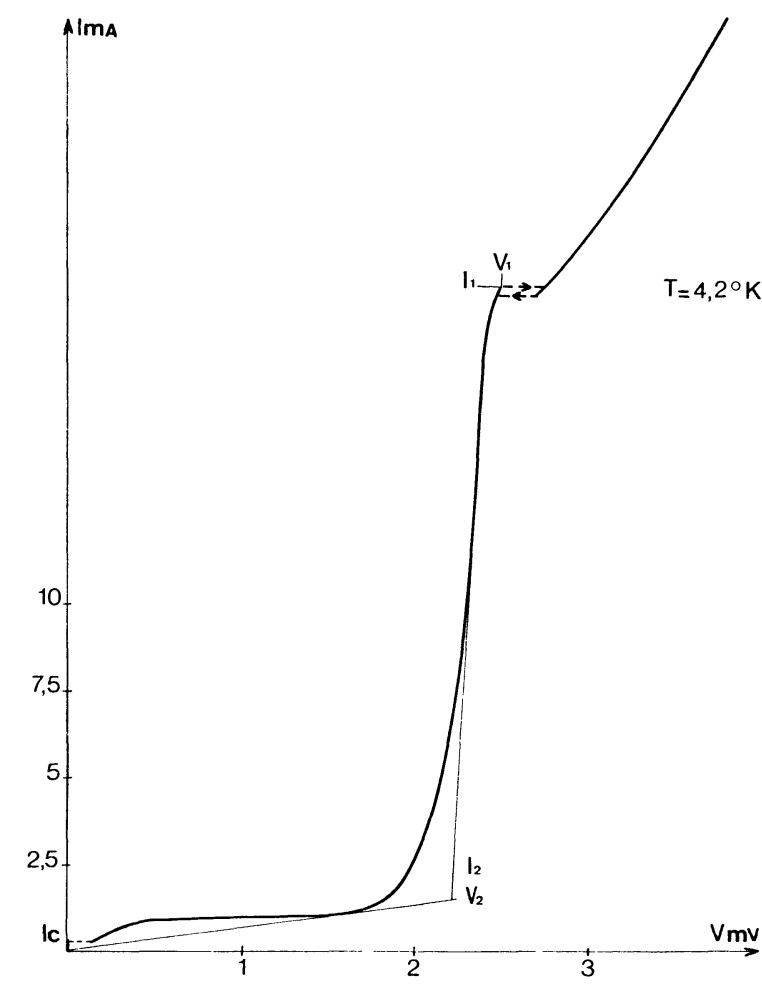

Fig. 4. - Caractéristique $I(V)$ de la jonction no $21 \mathrm{C}$ à barrière de germanium niobium-germanium-plomb avec environ $100 \AA$ d'épaisseur de germanium.

Sur le plan technologie, nous terminerons en notant qu'il est impossible de déposer un deuxième film de niobium, ceci essentiellement parce que l'énergie des particules incidentes détériore la barrière (oxyde ou germanium).

6. Conclusion. - D'abord, nous signalons que ce type de jonction utilisant un film de niobium comme base présente d'indéniables qualités de solidité et de stabilité dans le temps.

Cependant du fait du type capacitif de ces jonctions et de la faible conduction en parallèle qui existe (ceci à cause de leurs bonnes qualités) elles sont très sensibles aux parasites.

Ainsi on observe parfois, la jonction étant en cours de test, des modifications brutales de la caractéristique, se traduisant essentiellement par l'apparition de microcircuits en parallèle qui d'ailleurs n'affectent pas l'allure générale de la courbe.

\section{Bibliographie}

[1] Cardinne, P., Manhes, B., Morens, M., Journées de Technologie du Vide SFITV, Versailles mai 1972, 111-117.

[2] Hoel, L. S., Keller, W. H., Nordman, J. E., Scott, A. C., Solid State Electronics 5 (1972) 1167-1173.

[3] Cardinne, P., Nordman, J. E., 3e Colloque d'Aussois sur la Supraconductivité, avril 1973. Revue Phys. Appl. 8 (1973) 467.
[4] Gilabert, Romagnan, Guyon, C. R. Hebd. Séan. Acad. Sci. 21 (1970) 552-555.

[5] Townsend, P., Sutton, J., Phys. Rev. 128 (1962) 591595.

[6] Keller, W. H., Nordman, J. E., Phys. Lett. 36A (1971) 52.

[7] Schwidtal, J. Appl. Phys. 43 (1972) 202-208. 